\title{
Sistematização da assistência de enfermagem em Centro Obstétrico
}

\author{
Systematization of nursing care in the Obstetrical Center
}

Sistematización de la atención de enfermería en el Centro Obstétrico

\author{
Raquel Bezerra dos Santos', Karla da Silva Ramos" \\ 'Maternidade Jesus Nazareno. Faculdade da Associação Caruaruense de Ensino Superior, \\ Curso de Bacharelado em Enfermagem. Caruaru-PE, Brasil. \\ "Instituto de Medicina Integral Prof. Fernando Figueira. Faculdade Pernambucana de Saúde, \\ Curso de Enfermagem. Recife-PE, Brasil.
}

Submissão 24-09-2010 Aprovação: 04-01-2012

RESUMO

Trata-se de um estudo descritivo-exploratório, com abordagem quantitativa, que objetivou propor um protocolo para a Sistematização da Assistência de Enfermagem para parturientes no Centro Obstétrico de um hospital público em Recife-PE. Foi aplicado um instrumento semiestruturado a quarenta parturientes para a obtenção do histórico de enfermagem; a partir deste foram identificados os diagnósticos de enfermagem baseados na Classificação Internacional para a Prática de Enfermagem $\left(\right.$ CIPE $\left.^{\circ}\right)$, versão 1, e estabelecidos os respectivos resultados e intervenções de enfermagem. O protocolo consiste em duas etapas: a primeira é a consulta de enfermagem, que envolve a anamnese e exame físico, e a segunda é caracterizada pela identificação criteriosa dos diagnósticos de enfermagem, que direcionarão o planejamento da assistência de enfermagem para o atendimento individualizado às parturientes, com utilização de uma terminologia universal.

Descritores: Assistência de enfermagem; Diagnóstico de enfermagem; Enfermagem Obstétrica; Trabalho de parto.

\section{ABSTRACT}

This is a descriptive and exploratory study with a quantitative approach, aiming to propose a protocol for the systematization of nursing care to women in the process of giving birth in the Obstetrical Center of a public hospital in Recife, Pernambuco, Brazil. A semi-structured instrument was applied to forty women in the process of giving birth, in order to obtaining the nursing history; from which the nursing diagnoses were identified, having as a basis the International Classification for Nursing Practice $(\mathrm{ICNP})^{\circ}$, version 1, and their respective results and nursing interventions were established. The protocol consists in two stages: the first one is the nursing consultation, which involves the anamnesis and physical examination; and the second, involves the judicious identification of the nursing diagnoses, which will guide the planning of the nursing care to provide the individualized attention to women in the process of giving birth, using a universal terminology.

Key words: Nursing care; Nursing diagnosis; Obstetrical Nursing; Labor.

\section{RESUMEN}

Esto es un estudio descriptivo y exploratorio, con enfoque cuantitativo que objetivó proponer un protocolo para la sistematización de la atención de enfermería a las parturientes en el Centro Obstétrico de un hospital público en Recife, Pernambuco, Brasil. Fue aplicado un instrumento semiestructurado a cuarenta parturientes para la obtención del histórico de enfermería y, a partir de este, fueron identificados los diagnósticos de enfermería basados en la Clasificación Internacional de la Práctica de Enfermería (CIPE), versión 1, y establecidos los respectivos resultados y intervenciones de enfermería. El protocolo consiste en dos etapas: la primera es la consulta de enfermería, que envuelve la anamnesis y examen físico; la segunda es caracterizada por la identificación juiciosa de los diagnósticos de enfermería, que guiaran el planeamiento de la atención de enfermería para la atención individualizada a las parturientes, desde una terminología universal.

Palabras clave: Atención de Enfermería; Diagnóstico de Enfermería; Enfermería Obstétrica; Trabajo de Parto.

Extraído de Monografia da Residência de Enfermagem em Saúde da Mulher, Hospital Barão de Lucena. Recife-PE, 2009.

AUTOR CORRESPONDENTE Raquel Bezerra dos Santos E-mail: raquelbezerra@yahoo.com.br 


\section{INTRODUÇÃO}

O parto é uma experiência única, de significância psicológica, que pode deixar marcas positivas ou negativas dependendo da experiência vivenciada pela mulher ${ }^{(1)}$. É um processo, além de fisiológico, cheio de significados onde ela deve ser a protagonista desse acontecimento ${ }^{(2)}$.

Antigamente, o parto acontecia nos lares sendo acompanhado por parteiras que, embora não tivessem o conhecimento científico, eram detentoras de vasta experiência, e cuja conduta expectante respeitava o nascimento. Entretanto, a partir do século XX, o parto passou a ser realizado em ambiente hospitalar, submetendo a mulher ao modelo biomédico, onde o profissional vê o parto como processo patológico e utiliza a medicalização e outros procedimentos no parto de forma abusiva ${ }^{(2-3)}$.

Em 1922, foi fundada no Rio de Janeiro a Escola de Enfermagem Anna Nery, contemplando em seu currículo a arte de enfermagem na obstetrícia e ginecologia. A partir deste momento, a enfermeira começou a adquirir conhecimentos sobre o processo de parturição e, em 1988, a enfermeira obstetra foi inserida na assistência ao parto hospitalar, pela primeira vez, na tentativa de reduzir os índices de asfixia perinatal ${ }^{(2,4)}$. A Organização Mundial de Saúde (OMS) reconhece que a realização do parto normal pelo enfermeiro obstetra tem caráter menos intervencionista e sua técnica humanizada respeita a fisiologia do nascimento ${ }^{(5)}$. Além disso, seus conhecimentos permitem detectar e corrigir precocemente qualquer intercorrência e/ou distócia no trabalho de parto, assegurando, por participação ativa, que será mantida uma condução adequada pela equipe obstétrica.

Apesar da formação técnico-cientifica e da Resolução do Ministério da Saúde e do Conselho Federal de Enfermagem MS/COFEN - 223/99(6) acobertar ética e legalmente a atuação do enfermeiro obstetra a prestar assistência à mulher durante todo ciclo gravídico puerperal, ainda é pequeno o número de partos realizados e registrados por ele $\mathrm{e}^{(5)}$.

Na maioria das vezes, o enfermeiro presta uma assistência descontextualizada, tendo em vista que suas ações são voltadas apenas para o procedimento em si e não para a mulher, limitando-se apenas à prescrição médica para prestar assistência à parturiente. Além disso, no dia-a-dia do enfermeiro, existe o excesso de atividades burocráticas voltadas para o controle de material, gerenciamento do pessoal de enfermagem, registros e relatórios escritos de forma não sistematizada, caracterizando a execução de tarefas rotineiras, e revelando, assim, a falta de disponibilidade com a assistência( ${ }^{(7-8)}$.

O enfermeiro desenvolve habilidades técnico-científicas que favorecem a organização e sistematização do cuidado. A Enfermagem moderna utiliza os conhecimentos e procedimentos teoricamente organizados e reformulados para implementar a sistematização da assistência de enfermagem (SAE). A Resolução COFEN no 358/2009 considera que a SAE "organiza o trabalho profissional quanto ao método, pessoal e instrumento, tornando possível a organização do Processo de Enfermagem"(9).

O Processo de Enfermagem (PE) "é um instrumento metodológico que orienta o cuidado profissional da Enfermagem e a documentação da prática profissional”(9), aumentando a visibilidade e o reconhecimento profissional. O registro deve ser realizado formalmente em um instrumento que apresente o resumo dos dados coletados durante a consulta de enfermagem, os diagnósticos de enfermagem identificados a partir da consulta, as ações de enfermagem realizadas e os resultados alcançados ${ }^{(9)}$.

Existem alguns sistemas de classificação que favorecem a padronização da linguagem facilitando o desenvolvimento de pesquisas, do processo de ensino aprendizagem e promoção da cientificidade do cuidado. Destacam-se como sistemas de classificação a Taxonomia II da NANDA Internacional; a Classificação das Intervenções de Enfermagem (NIC); a Classificação dos Resultados de Enfermagem (NOC); o sistema de OMAHA (classificação para a saúde comunitária); o Sistema de Classificação de Cuidados Clínicos (CCC); e a Classificação Internacional para a Prática de Enfermagem (CIPE) , que foi desenvolvida com a unificação de vocabulários da Enfermagem, como a NANDA, NIC, NOC, e sistema da OMAHA, entre outros ${ }^{(10)}$.

A CIPE é um sistema de linguagem de enfermagem, unificado e universal, que integra as diversas classificações desenvolvidas ao longo do tempo, facilitando a melhoria da assistência ao cliente e permitindo a visibilidade da contribuição do enfermeiro na equipe multiprofissional. Em 1989, o Conselho de Representantes Nacionais aprovou o desenvolvimento do sistema da CIPE no $19^{\circ}$ Congresso Quadrienal do Conselho Internacional de Enfermagem, em Seul, na Coréia. A falta de um sistema de classificação de linguagem unificada foi o principal motivo para sua elaboração, visto que essa é condição essencial para a formulação de políticas de saúde, gerenciamento de custos, informatização dos serviços de saúde e controle do trabalho de enfermagem ${ }^{(11)}$.

Em dezembro de 1996, o Conselho Internacional de Enfermagem (CIE) publicou a Classificação Internacional para a Prática de Enfermagem - Versão Alfa: Um Marco Unificador, constituída pela Classificação dos Fenômenos de Enfermagem e pela Classificação das Intervenções de Enfermagem, a qual sofreu reformulações dando origem a CIPE $E^{\circledast}$ Versão Beta, divulgada em julho de 1999 durante as comemorações dos 100 anos do CIE. E em janeiro de 2002, foi apresentada a CIPE Versão Beta 2, que trazia a Classificação de Fenômenos de Enfermagem estruturada em oito eixos e a classificação das ações, em outros oito eixos ${ }^{(12)}$.

A partir da ampla utilização da CIPE ${ }^{\oplus}$ Versão Beta 2, percebeu-se a ausência da unificação da linguagem na enfermagem, o que gerou mais uma reformulação dando origem à CIPE ${ }^{\oplus}$ Versão 1 como uma tentativa de se evitar redundância e ambiguidade entre os termos a partir de uma estrutura de classificação simplificada organizada em apenas sete eixos originados após a unificação dos oito eixos da classificação dos fenômenos de enfermagem e dos oito, da classificação das ações de enfermagem existentes na CIPE ${ }^{\bullet}$ Versão Beta $2^{(11)}$.

Tendo em vista o exposto, este trabalho teve por objetivo propor um protocolo para atendimento sistematizado de enfermagem às parturientes no Centro Obstétrico de um hospital público na Cidade do Recife-PE.

\section{METODOLOGIA}

Trata-se de um estudo descritivo e exploratório com abordagem quantitativa, realizado no período de outubro de 2007 a 
janeiro de 2008. A pesquisa aconteceu no centro obstétrico de um hospital público estadual Amigo da Criança, na cidade do Recife-PE, o qual é referência em atendimento à gestante de alto risco, contudo também atende à gestante de baixo risco quando estas chegam em trabalho de parto avançado, sendo inviável sua transferência para outra maternidade de referência.

A população do estudo foi constituída por 40 parturientes admitidas no centro obstétrico da referida instituição, com diagnóstico médico de Gestação Única Tópica a Termo e Trabalho de Parto, sem nenhuma outra patologia associada a elas e idade superior a 18 anos.

A elaboração do protocolo foi dividida em três etapas. A primeira constituiu-se na coleta de dados a partir da identificação da parturiente no pré-parto e explicação sobre os objetivos da pesquisa. Após a assinatura do Termo de Consentimento Livre e Esclarecido (TCLE), foi iniciada a entrevista e coleta de dados no prontuário.

Foi utilizado um instrumento para a formulação do Histórico de Enfermagem. Este foi preenchido pela própria autora em dois momentos: através de entrevista direta com a parturiente durante o trabalho de parto e, posterior, coleta de dados no prontuário sobre a admissão da parturiente e registros do parto.

A partir daí, procedeu-se à segunda etapa, quando as informações do formulário foram digitadas em um banco de dados confeccionado especialmente para este fim. Após o levantamento de dados, foram identificados os diagnósticos de enfermagem. Para isso, foi feito um agrupamento das informações, seguido da utilização das orientações contidas na CIPE $^{\circledast}$ versão 1.0 para formulação dos diagnósticos e resultados de enfermagem utilizando-se os eixos foco (base para o planejamento da assistência) e julgamento.

A terceira etapa foi constituída pela elaboração das intervenções de enfermagem. Nesse momento, foi realizada uma pesquisa eletrônica da literatura utilizando-se as seguintes bases de dados: Literatura Latino-Americana e do Caribe em Ciências da Saúde (LILACS) e Scientific Electronic Library Online (SciELO). $\mathrm{O}$ acesso online aos artigos deu-se por meio do Centro Latino-Americano e do Caribe de Informações em Ciências da Saúde (Bireme). Utilizaram-se artigos publicados no período de 2000 a 2010, a partir dos descritores: Parto Humanizado e Enfermagem Obstétrica. A pesquisa resultou no encontro de nove artigos, os quais abordavam práticas de cuidado durante o trabalho de parto e parto. Após o estudo dos artigos, realizou-se a construção das intervenções de enfermagem incluindo-se um termo do eixo ação e termos de qualquer outro eixo, com exceção ao eixo julgamento.

A pesquisa foi aprovada pelo Comitê de Ética em Pesquisa (CEP) do Hospital Agamenon Magalhães sob $n^{\circ}$ de Protocolo 259/2006, conforme a Resolução n 196/96 da Comissão Nacional de Ética e Pesquisa em Seres Humanos, do Ministério da Saúde, que estabelece normas e diretrizes regulamentadoras de pesquisa envolvendo seres humanos.

\section{RESULTADOS E DISCUSSÃO}

No pré-parto, a proposta de assistência à parturiente consiste inicialmente em uma consulta de enfermagem. A consulta de enfermagem é momento fundamental para investigação das necessidades da parturiente, subsidiando criteriosamente as demais etapas do Processo de Enfermagem. O formulário para elaboração do Histórico de Enfermagem foi concebido a partir da necessidade de identificação dos dados obstétricos da parturiente para facilitar a SAE. O instrumento para consulta de enfermagem contempla os dados de identificação da parturiente, os dados relacionados à gestação, exame físico da parturiente, exames laboratoriais e dados do parto (Quadro 1).
Dados de identificação

Nome:

Data de nascimento:

Procedência:

Estado civil:

Ocupação:

Escolaridade:

Dados relacionados à gestação

Número de consultas de pré-natal

Data da última menstruação:

Idade gestacional:

Gestação: Partos: $\quad N^{\circ}$ de Partos normais: $\quad N^{\circ}$ de Partos cesariana: $\quad$ Aborto:

Presença de alguma patologia durante a gravidez: ( ) Não Sim ( ), Qual?

Recebeu orientação quanto ao trabalho de parto durante o pré-natal? ( ) Não Sim ( )

\section{Exame físico da parturiente}

SSVV: Temperatura:__ _ ${ }^{\circ}$; Pulso:___ bpm; Frequência Respiratória:___rpm; Pressão Arterial:

Exame das mamas:

Exame abdominal:

AFU - _ cm; BCF: _ _ bpm; Dinâmica Uterina:

Toque vaginal:

Observação:

Queixas:

\section{Exames laboratoriais}

Classificação sanguínea e fator Rh:

VDRL:

HIV:

Dados do parto

Tipo de parto:

Descrição do parto:

Observação:

Quadro 1 - Consulta de enfermagem à parturiente no pré-parto e sala de parto 
Após a consulta de enfermagem, deve ser iniciada a segunda etapa do protocolo que é a identificação dos diagnósticos de enfermagem. Um formulário específico foi elaborado a partir dos dados coletados com as participantes da pesquisa. O mesmo contempla sete diagnósticos de enfermagem com suas respectivas intervenções e resultados esperados (Quadro 2).

A investigação sobre os dados contidos no instrumento para a consulta de enfermagem auxilia a tomada de decisão quanto às condutas a serem tomadas pelo enfermeiro obstetra e sua equipe. O protocolo orienta as intervenções de enfermagem na perspectiva da promoção e da facilitação de um parto saudável, fisiológico, sem intervenções desnecessárias a partir da identificação dos diagnósticos de enfermagem.

A busca de qualidade deve ser um processo contínuo subsidiado pela competência técnico-científica e comprometimento do profissional. A SAE permite que o enfermeiro obstetra se concentre no campo de conhecimento peculiar à enfermagem em busca do nível de qualidade compatível com as necessidades da parturiente ${ }^{(13)}$.

É na sala de pré-parto que o medo de morrer, medo de perder o filho e o medo da dor se intensificam na mulher, e podem dominar a experiência da parturição negativamente, especialmente quando associados a outros sentimentos ou fatores como o stress, ansiedade, fadiga, tensão, frio, fome, ambiente estranho, desamparo social e afetivo ${ }^{(14)}$.

Foi a partir do relato das parturientes sobre as sensações que estavam vivenciando durante o trabalho de parto que foram identificados os diagnósticos de enfermagem, como: medo do trabalho de parto, fadiga presente, contração uterina normal e dor de trabalho de parto presente. $E$, mediante os procedimentos a que foram submetidas no parto, outros diagnósticos foram elaborados: expulsão uterina fetal sem distócias ou intercorrências, laceração do períneo ou vagina e corte na região médio-lateral do períneo.

Uma das finalidades do enfermeiro obstetra é proporcionar um ambiente calmo que reduza a ansiedade e o medo nas mulheres em trabalho de parto, além de ofertar técnicas para o alívio da dor como massagem lombar, deambulação, posturas variadas durante o trabalho de parto e parto, hidratação, alimentação, métodos de respiração para minimizar o desconforto do processo parturitivo, e banho de imersão. Todos esses fatores são alternativas para o conforto da parturiente que podem aliviar a dor sem interferir na progressão do trabalho de parto ${ }^{(14)}$.

\begin{tabular}{|c|c|c|}
\hline Diagnóstico de Enfermagem & Resultados Esperados & Intervenções de Enfermagem \\
\hline Contração uterina normal & $\begin{array}{l}\text { Contração uterina } \\
\text { em progressão }\end{array}$ & $\begin{array}{l}\text { Avaliar a dinâmica uterina registrando sua frequência, duração e intensidade; } \\
\text { Monitorar o batimento cardíaco fetal (BCF) antes, durante e após as contrações para } \\
\text { identificar presença de desacelerações intrapélvicas; } \\
\text { Informar ao profissional médico alterações no BCF e/ou nas contrações uterinas. }\end{array}$ \\
\hline Dor de trabalho de parto & $\begin{array}{l}\text { Dor de trabalho de } \\
\text { parto diminuída }\end{array}$ & $\begin{array}{l}\text { Encorajar a deambulação durante o trabalho de parto; } \\
\text { Incentivar as posições verticais; } \\
\text { Ensinar técnicas de respiração e relaxamento; } \\
\text { Promover banho de chuveiro morno e massagem lombossacral; } \\
\text { Registrar a evolução do trabalho de parto em partograma. }\end{array}$ \\
\hline Medo do trabalho de parto & Medo diminuído & $\begin{array}{l}\text { Conversar usando uma abordagem calma e segura; } \\
\text { Explicar o processo de trabalho de parto e parto esclarecendo suas dúvidas; } \\
\text { Permitir presença de acompanhante conforme desejo da parturiente; } \\
\text { Implementar o toque afetivo para acalmá-la realizado pelo enfermeiro ou } \\
\text { acompanhante escolhido pela parturiente; } \\
\text { Oferecer terapia com música. }\end{array}$ \\
\hline Fadiga & Fadiga melhorada & $\begin{array}{l}\text { Orientar a parturiente quanto à forma de fazer relaxamento muscular para que ela } \\
\text { descanse entre uma contração e outra. }\end{array}$ \\
\hline $\begin{array}{l}\text { Expulsão uterina fetal sem } \\
\text { distócias ou intercorrências }\end{array}$ & $\begin{array}{l}\text { Expulsão uterina } \\
\text { realizada }\end{array}$ & $\begin{array}{l}\text { Deixar passar lentamente a cabeça do feto; } \\
\text { Proteger o períneo durante o nascimento; } \\
\text { Clampear e cortar o cordão umbilical após o término das pulsações; } \\
\text { Verificar integridade da placenta após delivramento; } \\
\text { Colocar o recém-nascido junto ao seio materno para estimular o aleitamento e } \\
\text { liberação de ocitocina. }\end{array}$ \\
\hline $\begin{array}{l}\text { Laceração do períneo } \\
\text { ou vagina }\end{array}$ & $\begin{array}{l}\text { Prevenção de } \\
\text { contaminação } \\
\text { da laceração }\end{array}$ & $\begin{array}{l}\text { Examinar o canal vaginal após o delivramento fisiológico da placenta para verificar } \\
\text { presença de lacerações; } \\
\text { Realizar correção de laceração após administração de anestésico local; } \\
\text { Orientar sobre higiene na região vulvoperineal e uso de roupas limpas; }\end{array}$ \\
\hline $\begin{array}{l}\text { Corte na região médio-lateral } \\
\text { do períneo }\end{array}$ & $\begin{array}{l}\text { Prevenção de } \\
\text { contaminação do } \\
\text { corte na região } \\
\text { médio-lateral do } \\
\text { períneo }\end{array}$ & $\begin{array}{l}\text { Fazer infiltração de anestésico local, se necessário; } \\
\text { Fazer corte na região médio lateral do períneo (episiotomia), se necessário; } \\
\text { Realizar episiorrafia; } \\
\text { Fazer toque retal para garantir a integridade tissular; } \\
\text { Orientar sobre higiene na região vulvoperineal e uso de roupas limpas. }\end{array}$ \\
\hline
\end{tabular}

Quadro 2 - Planejamento da assistência de enfermagem no pré-parto e sala de parto 
Atualmente, as posições mais indicadas durante o trabalho de parto são as verticalizadas por permitir melhor circulação feto-placentário, menos dificuldade na hora dos puxos, redução das dores, diminuição das lacerações vaginais e perineais e período expulsivo mais rápido ${ }^{(15)}$.

Estudos referem que o pré-natal tem importância fundamental no preparo da mulher para o trabalho de parto ${ }^{(16)}$. No entanto, verifica-se, na prática, que faltam informações fundamentais para que elas vivenciem esse momento com segurança. O desconhecimento da mulher sobre o processo fisiológico do parto dificulta seu protagonismo, tomada de decisões e escolhas conscientes sobre as intervenções a qual estará se submetendo ${ }^{(17)}$.

Um dos procedimentos mais comuns na Obstetrícia, que tem enfoque intervencionista subtraindo da mulher parturiente a possibilidade de vivenciar o parto como um processo fisiológico e fortalecedor de sua autonomia, é a episiotomia ${ }^{(18)}$. A episiotomia é uma incisão cirúrgica na região vulvar, com indicação obstétrica, para impedir ou diminuir o trauma dos tecidos do canal do parto. Em número, é superado apenas pelo corte e pinçamento do cordão umbilical. No entanto, seu uso é recomendado em apenas 15 a 30\% dos casos. Comumente o enfermeiro obstetra respeita os critérios para sua realização e opta por uma atitude menos intervencionista ${ }^{(15)}$.

$\mathrm{Na}$ tentativa de se evitar práticas iatrogênicas durante o trabalho de parto, a OMS recomenda a utilização do partograma para acompanhamento do trabalho de parto ${ }^{(18)}$. Esse instrumento consiste na representação gráfica do trabalho de parto através de um traçado feito no início da fase ativa do trabalho de parto, chamada de linha de alerta, e outro traçado, quatro horas após o primeiro, determinando a linha de ação. Isso facilitará a avaliação do trabalho de parto em eutócico ou distócico, tendo em vista que ele avalia a dilatação cervical, descida da apresentação e, ainda, permite o registro dos batimentos cardiofetais, dinâmica uterina, medicações administradas, entre outros ${ }^{(19)}$.

Estudos mostraram que técnicas como deambulação, movimentação e banho de aspersão ou imersão são cada vez mais utilizados à medida que o trabalho de parto progride para as zonas II e III do partograma, mostrando sua importância como critério para modificação de condutas obstétricas ${ }^{(19)}$.

O papel do enfermeiro obstetra é ajudar nas forças naturais do parto, criando condições mais favoráveis para o nascimento, vivenciando a ciência, a natureza e a ética, promovendo, assim, modificações de comportamento de acordo com as respostas da mulher, fazendo com que ela, ao parir, consiga atingir o mais alto grau de satisfação ${ }^{(20)}$.

Um protocolo de enfermagem nos setores de pré-parto e sala de parto estimulará a efetivação do enfermeiro obstetra no acompanhamento do trabalho de parto e parto a partir da implementação de técnicas como deambulação, utilização do toque afetivo, musicoterapia dentre outras intervenções já descritas. Desta forma, o enfermeiro deixa de se basear apenas na prescrição médica para realizar seus cuidados e começa a utilizar seu senso crítico para o planejamento da assistência evidenciando sua autonomia e favorecendo o reconhecimento profissional pelos membros da equipe.

\section{CONSIDERAÇÕES FINAIS}

A visão holística do enfermeiro obstetra associada ao Processo de Enfermagem, favorece uma assistência individualizada à parturiente, fundamentada no conhecimento científico, fazendo com que ela sinta-se parte de um processo natural, acompanhando o ritmo do seu próprio corpo.

A partir deste estudo foi possível propor um protocolo visando o desenvolvimento da Sistematização da Assistência de Enfermagem no pré-parto e sala de parto de um Hospital Público na cidade do Recife, uma vez que a Resolução do COFEN n 358/99 afirma que: "a implementação da Sistematização da Assistência de Enfermagem deve acontecer em toda instituição de saúde, pública e privada"(9).

É nesse contexto que a incorporação da $\mathrm{CIPE}^{\circledast}$ às atividades do enfermeiro o levará a obter melhores resultados na qualidade de sua assistência no centro obstétrico, uma vez que ele estará utilizando uma terminologia reconhecida internacionalmente facilitando a comunicação entre seus pares.

\section{REFERÊNCIAS}

1. Lopes RCS, Donelli TS, Lima CM, Piccinini CA. O antes e o depois: expectativas e experiências de mães sobre o parto. Psicol Reflex Crit 2005;18(2):247-54.

2. Zveiter M, Progianti JM, Vargens OMC. O trauma no parto e nascimento sob a lente da enfermagem obstétrica. Pulsional Rev Psicanál 2005;182(6):86-92.

3. Sodré TM, Lacerda, RA. O processo de trabalho na assistência ao parto em Londrina-PR. Rev Esc Enferm USP 2007;41(1):82-9.

4. Mouta RJO, Progianti. Estratégias de luta das enfermeiras da Maternidade Leila Diniz para implantação de um modelo humanizado de assistência ao parto. Texto \& Contexto Enferm 2009;18(4):731-40.

5. Barbosa PG, Carvalho GM, Oliveira LR. Enfermagem obstétrica: descobrindo as facilidades e dificuldades do especialista nesta área. Mundo da Saúde 2008;32(4):458-65.

6. Conselho Federal de Enfermagem [homepage na internet]. Resolução COFEN n²23/ 99. Dispõe sobre a atuação de Enfermeiros na Assistência à Mulher no Ciclo Gravídico Puerperal. [Acesso em 27 de maio 2005]. Disponível em: http://www.portalcoren-rs.gov.br/web/resoluca/r223.htm.

7. Moura ACF, Rabelo CBM; Sampaio MRFB. Prática profissional e metodologia assistencial dos enfermeiros em hospital filantrópico. Rev Bras Enferm 2008;61(4):476-81.

8. Truppel TC, Meier MJ, Calixto RC, Peruzzo SA, Crozeta K. Sistematização da Assistência de Enfermagem em Unidade de Terapia Intensiva. Rev Bras Enferm 2009;62(2):221-7.

9. Conselho Federal de Enfermagem [homepage na internet]. 
Resolução COFEN no 358/99. Dispõe sobre a Sistematização da Assistência de Enfermagem e a implementação do Processo de Enfermagem em ambientes, públicos ou privados, em que ocorre o cuidado profissional de Enfermagem. [Acesso em 12 de set 2012]. Disponível em: http:// www.portalcofen.gov.br/sitenovo/node/4384.

10. Fuly PSC, Leite JL, Lima SBS. Correntes de pensamentos nacionais sobre Sistematização da Assistência de Enfermagem. Rev Bras Enferm 2008;61(6):883-7.

11. Conselho Internacional de Enfermagem. Classificação Internacional para a Prática de Enfermagem $\left(\mathrm{CIPE}^{\circledR}\right)$ - Versão 1. Genebra, Suíça: 2006.

12. Bittencourt GKGD, Beserra PJF, Nóbrega MML. Assistência de enfermagem a paciente com lúpus eritematoso sistêmico utilizando a CIPE. Rev Gaúch Enferm 2008;29(1):26-32.

13. D'Inocenzo M, Adami NP, Cunha ICKO. O movimento pela qualidade nos serviços de saúde e enfermagem. Rev Bras Enferm 2006;59(1):84-8.

14. Davim RMB, Torres GV, Dantas JC, Melo ESM, Paiva $\mathrm{CP}$, Vieira D, et al. Banho de chuveiro como estratégia não farmacológica no alivio da dor de parturientes Rev. eletrônica Enferm. [periódicos na internet].2008 [acesso em 20 jan 2010];10(3):600-9 Disponível em: http://www. fen.ufg.br/revista/v10/n3/v10n3a06.htm

15. Mouta RJO, Pilloto DTS, Vargens OMC, Progianti JM. Relação entre posição adotada pela mulher no parto, integridade perinel e vitalidade de recém-nascido. Rev Enferm UERJ 2008;16(4):472-76.

16. Diniz CSG. Humanização da assistência ao parto no Brasil: os muitos sentidos de um movimento. Ciênc e Saúde Coletiva 2005; 10(3):627-37.

17. Gama AS, Giffin KM, Tuesta AJA, Barbosa GP, D'Orsi E. Representações e experiências das mulheres sobre a assistência ao parto vaginal e cesárea em maternidades pública e privada. Cad Saúde Pública 2009;25(11):2480-8.

18. Organização Mundial de Saúde. Maternidade Segura. Assistência ao Parto Normal: um guia prático. Genebra: 1996.

19. Rocha IMS, Oliveira SMJV, Schneck CA, Riesco MLG, Costa ASC. O partograma como instrumento de análise da assistência ao parto. Rev Esc Enferm USP 2009;43(4):880-8.

20. Davim RMB, Bezerra LGM. Assistência à parturiente por enfermeiras obstétricas no Projeto Midwifery: um relato de experiência. Rev Latinoam Enferm 2002;10(5):727-32. 